\title{
Chromatographic Alignment of LC-MS and LC-MS/MS Datasets by Genetic Algorithm Feature Extraction
}

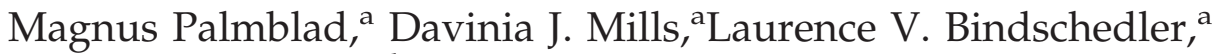 \\ and Rainer Cramer ${ }^{a, b}$ \\ a The BioCentre, The University of Reading, Whiteknights, Reading, United Kingdom \\ b Department of Chemistry, The University of Reading, Reading, United Kingdom
}

Liquid chromatography-mass spectrometry (LC-MS) datasets can be compared or combined following chromatographic alignment. Here we describe a simple solution to the specific problem of aligning one LC-MS dataset and one LC-MS/MS dataset, acquired on separate instruments from an enzymatic digest of a protein mixture, using feature extraction and a genetic algorithm. First, the LC-MS dataset is searched within a few ppm of the calculated theoretical masses of peptides confidently identified by LC-MS/MS. A piecewise linear function is then fitted to these matched peptides using a genetic algorithm with a fitness function that is insensitive to incorrect matches but sufficiently flexible to adapt to the discrete shifts common when comparing LC datasets. We demonstrate the utility of this method by aligning ion trap LC-MS/MS data with accurate LC-MS data from an FTICR mass spectrometer and show how hybrid datasets can improve peptide and protein identification by combining the speed of the ion trap with the mass accuracy of the FTICR, similar to using a hybrid ion trap-FTICR instrument. We also show that the high resolving power of FTICR can improve precision and linear dynamic range in quantitative proteomics. The alignment software, msalign, is freely available as open source. (J Am Soc Mass Spectrom 2007, 18, 1835-1843) (C) 2007 American Society for Mass Spectrometry

I $\mathrm{n}$ proteomics it is often useful to compare or merge data from different samples, or data acquired using different types of instrumentation-sometimes even from different laboratories. One example is an approach to biomarker discovery comparing features in mass spectrometry (MS) or liquid chromatography-mass spectrometry (LC-MS) datasets [1]. Potential biomarkers may subsequently be targeted for identification using tandem mass spectrometry (MS/MS). Another potential application is the combination of MS/MS data from a fast ion trap mass spectrometer with accurate MS data from a TOF or FTICR instrument to improve confidence in peptide and protein identification. High resolving power may also improve precision in quantitative proteomics by reducing interferences between peptides of similar $m / z$. This kind of data is readily produced in hybrid ion trap-FTICR instruments [2], but could also be constructed by merging ion trap LCMS/MS and FTICR datasets acquired separately in simpler instruments.

Direct comparison or combination of LC-MS or LCMS/MS datasets using all available information, including retention times, is challenging due to the rela-

Address reprint requests to Dr. M. Palmblad, The University of Reading, Whiteknights, P.O. Box 221, Reading RG6 6AS, United Kingdom.E-mail: n.m.palmblad@reading.ac.uk tively poor reproducibility of liquid separation methods, particularly in split-flow systems lacking flow rate or temperature control. However, time-dependent LC-MS datasets can be aligned by time warping, using some form of dynamic programming [3-7] or statistical methods [8]. Recently, Jaitly et al. [9] described a successful technique for aligning LC-MS and LCMS/MS datasets, including LC-MS to LC-MS/MS, through dynamic time warping by fitting piecewise functions to common features (observed peptides) in the datasets. The authors noted that this procedure is sensitive to outliers, such as incorrectly matched features or peptide ions. This problem could potentially be overcome with a more robust regression method, such as least median of squares regression [10]. Jaitly and coworkers solved the problem using a two-pass algorithm, where in the first pass a piecewise linear function was fitted to the matched features. The preliminary alignment was then used to recalibrate the FTICR mass spectra, providing a filter to remove false matches. After removing features considered as being too far from the exact mass of the identified peptide after recalibration, the remaining corrected features were realigned by cubic spline regression.

We have developed and implemented a comparatively simple, single-pass and inherently robust method for the special case of aligning one LC-MS dataset with 
one LC-MS/MS dataset using genetic algorithm (GA) feature extraction and piecewise linear functions. The robustness comes from a particular GA fitness function, which ignores outliers as long as there are sufficiently more correctly than incorrectly matched peptides. The program takes as input any LC-MS data in mzXML format [11] and the results of a database search from LC-MS/MS data in pepXML format [12]. The use of these public and open data formats is advantageous, as data from almost any pair of instruments can be combined using any of the common peptide/protein identification search engines. Utilities for converting data acquired on most commercial mass spectrometers to mzXML are freely available, as well as pepXML converters for search results, from Mascot, Sequest! and! X!! Tandem! (http:/ / tools.proteomecenter. org/software.php).

To evaluate and illustrate the alignment method, we applied it to bottom-up protein identification in yeast, combining LC-MS/MS data from an ion trap with accurate precursor ion masses from an FTICR mass spectrometer. We attempted to produce data of similar quality to the ion trap-FTICR data used by Andreev et al.![13]!for! relative!quantitation! using! ${ }^{15} \mathrm{~N}$ !labeling.!We labeled Arabidopsis thaliana plants metabolically with ${ }^{15} \mathrm{~N}$ and used the chromatographic alignment to combine ion trap LC-MS/MS and FTICR LC-MS data. We then compared the precision in relative protein abundance measurements with that achieved using ion trap data alone.

\section{Experimental}

\section{Protein Sample Preparation}

Saccharomyces cerevisiae diploid strain BY4743 was grown on YPD agar (Sigma, Gillingham, UK) plates at $30{ }^{\circ} \mathrm{C}$ for $48 \mathrm{~h}$. Two colonies were inoculated in $20 \mathrm{~mL}$ YPD media and grown overnight at $30^{\circ} \mathrm{C}$, reaching an o.d. ${ }_{600}$ of 1.45 . Ten $\mathrm{mL}$ from the overnight culture was transferred into $50 \mathrm{~mL}$ fresh medium and incubated for $2 \mathrm{~h}$ until reaching log-phase with o.d. $_{600}=0.5$. Yeast cells were harvested by centrifugation at $3000 \times g$ for 10 min at $4{ }^{\circ} \mathrm{C}$. The pellet $(0.44 \mathrm{~g})$ was resuspended in 2.5 $\mathrm{mL}$ YeastBuster reagent (Merck, Nottingham, UK) containing 1\% 100X tris(hydroxypropyl)phosphine solution, followed by the addition of $2.5 \mu \mathrm{L}$ Benzonase nuclease (Merck) and $5 \mu \mathrm{L}$ protease inhibitor cocktail (Merck). The mixture was shaken for $20 \mathrm{~min}$ at room temperature. Insoluble cell debris was then removed by centrifugation at $16,000 \times \mathrm{g}$ for $40 \mathrm{~min}$ at $4{ }^{\circ} \mathrm{C}$ and the supernatant stored at $-80^{\circ} \mathrm{C}$ until digestion with trypsin. The yeast proteins $(20 \mu \mathrm{g} ; 50 \mu \mathrm{L})$ were digested in solution. All chemicals were from Sigma unless otherwise stated. The cystines were reduced with the addition of $160 \mu \mathrm{L} 10 \mathrm{mM}$ DTT in $25 \mathrm{mM}$ ammonium bicarbonate $(\mathrm{ABC})$ and incubation at $56^{\circ} \mathrm{C}$ for $45 \mathrm{~min}$. The samples were then vacuum dried (Concentrator 5301; Eppendorf, Cambridge, UK). Cysteines were al-
Table 1. Chromatographic gradients as functions of time (in minutes)

\begin{tabular}{lrrr}
\hline \multicolumn{2}{c}{ Gradient 1 } & \multicolumn{2}{c}{ Gradient 2 } \\
\hline \hline \multicolumn{1}{c}{ Time } & $\%$ B & Time & $\%$ B \\
\hline \hline 0 & 0 & 0 & 0 \\
5 & 0 & 5 & 0 \\
90 & 40 & 155 & 40 \\
105 & 55 & 185 & 55 \\
106 & 100 & 187 & 100 \\
126 & 100 & 212 & 100 \\
127 & 0 & 215 & 0 \\
145 & 0 & 240 & 0 \\
\hline
\end{tabular}

kylated by adding $160 \mu \mathrm{L} 50 \mathrm{mM}$ iodoacetamide in 25 $\mathrm{mM} A B C$, followed by incubation for $1 \mathrm{~h}$ at room temperature, after which the samples were dried under vacuum. Sequencing grade porcine trypsin $(0.4 \mu \mathrm{g}$ in $160 \mu \mathrm{L} 25$ mM ABC; Promega, Southampton, UK); was added and digestion took place at $37^{\circ} \mathrm{C}$ for $24 \mathrm{~h}$. The digestion was quenched with the addition of $1.5 \mu \mathrm{L}$ of $10 \%$ TFA.

The combination of ion trap LC-MS/MS and FTICR LC-MS in quantitative proteomics was demonstrated in a comparison of uniformly ${ }^{15} \mathrm{~N}$-labeled Arabidopsis thaliana plants subjected to oxidative stress with untreated controls. This study will be published in detail elsewhere. The oxidative stress was induced by spraying 6-wk old plants with hydrogen peroxide, and the plants were harvested $40 \mathrm{~h}$ after the treatment. Total protein extracts were separated by SDS-PAGE, the gel subsequently cut into $252-\mathrm{mm}$ slices and the proteins digested and run on the ion trap LC-MS/MS and FTICR LC-MS!systems!in! parallel!using!Gradient!1!(Table!1).

\section{Liquid Chromatography-Mass Spectrometry}

Peptides from tryptic digests were reconstituted in $0.5 \%$ TFA, separated, and analyzed by LC-MS/MS using an Ultimate LC system (Dionex/LC Packings, Amsterdam, The Netherlands) coupled to an HCT ion trap (Bruker Daltonics, Bremen, Germany) or LC-MS using an identical LC system coupled to an Apex-Q FTICR mass spectrometer (Bruker Daltonics). Both LC systems were configured with a $10 \mathrm{~mm} \mathrm{C}_{18}$ trap column (Dionex) and a $15 \mathrm{~cm} \times 75 \mu \mathrm{m}$ PepMap $\mathrm{C}_{18}$ analytical column (Dionex) with $3.5 \mu \mathrm{m}$ particles. The performance and robustness of the algorithm was verified for chromatographic gradients of lengths from 100 to $720 \mathrm{~min}$. In the data presented here, one of two binary gradients was used! (Table! 1).! All! gradients! were! formed! from! 2\% acetonitrile (ACN) (A) and 90\% ACN (B), both with $0.1 \%$ formic acid, using a flow rate over the column of 250 to $300 \mathrm{~nL} / \mathrm{min}$ in the split-flow LC systems. The columns were equilibrated for at least 15 min in Buffer A before loading and desalting on the trap column for $5 \mathrm{~min}$, followed by injection onto the analytical column. The LC systems were controlled through HyStar version 3.1 (Bruker). The HCT and FTICR were controlled 
from esquireControl 5.3 and apexControl 1.0, respectively. Mass spectra were acquired in the HCT from $\mathrm{m} / z 200$ to 2000 using parameters optimized at $\mathrm{m} / \mathrm{z} 900$ with the trap ion charge control set at 100,000 and a maximum acquisition time of $150 \mathrm{~ms}$, averaging three scans per spectrum. The three most abundant ions were selected for MS/MS but dynamically excluded for $30 \mathrm{~s}$ after having been selected twice. FTICR LC-MS data were acquired from $m / z 280$ to 2500 with alternating MS and MS/MS without averaging (4.5 s/spectrum) with! Gradient! 1! (Table! 1)! or! averaging! 4! scans! (13.7 s/spectrum) with Gradient 2. The limited FTICR MS/MS data were not used as the method was designed to be generally applicable to LC-MS and LCMS/MS datasets.

DataAnalysis 3.3 (Bruker) was used to produce peak lists in Mascot generic format (MGF) from LC-MS/MS data. These MGF files were searched with Mascot 2.1 (Matrix Science, London, UK) using a peptide and MS/MS tolerance of $0.4 \mathrm{Da}$. The resulting files converted! to! pepXML! using! Mascot2XML! [14].! The! raw FTICR LC-MS data were converted to mzXML using compassXport (Bruker) for alignment using common peptides confidently identified by LC-MS/MS in the ion trap and accurate mass from the FTICR LC-MS data. The resulting alignment of the two datasets was then used to retrieve precursor ion masses from the FTICR data to replace the values in the original MGF file (leaving the MS/MS data from the ion trap unchanged). This hybrid dataset was then submitted to Mascot with 5 ppm peptide and 0.4 Da MS/MS tolerance. The peptide tolerance was chosen to include $>95 \%$ of the parent ion measurements, based on previous analysis of the distribution of mass measurement errors in this FTICR! instrument! [15].! The! most! intense! peak! in! the FTICR data within the mass measurement uncertainty of the ion trap in a narrow range around the aligned retention time was chosen as the single and best match in the FTICR data, the rationale being that this is most likely the peptide whose fragment ions will dominate the ion trap MS/MS spectrum.

The! Integr8![16]!S. cerevisiae (20070306)! and!A. thaliana (20070220) FASTA files were searched for yeast and Arabidopsis data, respectively, with trypsin specificity, carbamidomethylation as fixed modification, no variable modifications, and allowing one missed trypsin cleavage site. The HCT LC-MS/MS datasets were also converted to mzXML using compassXport for visual inspection of datasets and their alignment. The peptide and protein identifications in the hybrid datasets were also combined with ion trap-only datasets to include peptides that did not transfer to the hybrid dataset. The false positive rate in the Arabidopsis study was estimated by searching a random database generated by make_random! (http://www.ms-utils.org/make_random.html).! This! random! database! has! the! same! size, amino acid, tryptic peptide, and protein size distribution as the real $A$. thaliana database. All software was written in $C$ using publicly available libraries and run
under!Microsoft!Windows!in!the!Cygwin![17]!environment.

\section{Theory}

Most methods for chromatographic alignment operate on raw data and typically assume the datasets are of similar type and dimensionality. Low-resolution LCMS/MS data from an ion trap mass spectrometer can be aligned with high-resolution LC-MS data from an FTICR mass spectrometer by first searching a protein database! with! the! LC-MS/MS! data! [9].! The! exact! $m / z$ values of confidently identified peptides are then matched with corresponding extracted ion chromatogram maxima in the FTICR dataset. The problem is then reduced to curve fitting to a relatively small number of such discrete matches.

Feature extraction is a general term for methods producing simplified but accurate descriptions of large datasets, such as in image analysis. Examples of common challenges in feature extraction include the recognition of spatial features in the presence of noise or accurately describing main trends in time series data without fitting to individual time points. In reality, multiple processes or phenomena often contribute to the data and the overall pattern is not always easy to describe with a single mathematical function. A more general approach is to use piecewise functions, i.e., functions that are defined on a sequence of intervals. The functions in the different intervals may be completely different, but linear segments and splines are the most commonly used, as in piecewise linear or spline regression. A one-dimensional piecewise linear function $y(x)$ has the general form

$$
y(x)=\left\{\begin{array}{c}
k_{1} x+m_{1}, x_{0} \leq x<x_{1} \\
k_{2} x+m_{2}, x_{1} \leq x<x_{2} \\
\vdots \\
k_{n} x+m_{n}, x_{n-1} \leq x<x_{n}
\end{array}\right.
$$

where the $x_{0} \ldots x_{n}$ or $\left(x_{0}, y\left(x_{0}\right)\right) \ldots\left(x_{n}, y\left(x_{n}\right)\right)$ are commonly referred to as nodes or breakpoints. There are several reasons why piecewise functions are a natural choice for alignment of LC datasets. In the electronically programmed solvent gradients driving chromatographic separations, the fractions of different solvents are nearly always piecewise linear functions of time. The gradient used for LC-MS/MS may of course be different from the gradient used for LC-MS if these systems are independently optimized. Due to the mechanical nature of the solvent delivery systems in LC, the flow rate or mobile phase composition may change abruptly, for instance if the back pressure over the column changes due to clogging, thereby changing the flow rate in split-flow systems lacking flow sensor feedback. The pressure can also drop abruptly due to formation of gas bubbles. For these and other reasons, singularities are expected when aligning LC-MS datasets. When $k_{1} \ldots k_{n}$ 
$m_{1} \ldots m_{n}, x_{0} \ldots x_{n}$ and $n$ are all variable, eq 1 describes a very large set of functions, even with a moderate limit on $n$ and requiring the function to be continuous. The challenge with piecewise functions is finding the breakpoints and how many breakpoints produce the most realistic fit to the data.

\section{Genetic Algorithm}

Genetic algorithms (GA) are a class of algorithms inspired by biological evolution suitable for finding approximate solutions to global optimization problems, particularly when searching a very large space of possible solutions. The GA uses all or a subset of computer analogues to biological inheritance, mutation, selection, and recombination, by encoding candidate solutions in a form suitable for applying these operators. These representations of the candidate solutions are called chromosomes. The algorithm also requires a function for evaluating the fitness of an individual chromosome. This fitness function may be any function taking a chromosome as argument and returning a scalar value, without regard to differentiability. Starting from a randomly generated set of chromosomes and a fitness function, the GA iteratively selects the fittest candidate solutions in each generation and lets them reproduce, applying mutation and recombination to the chromosome representation of the solutions, while keeping the population size constant. This process eventually converges to the global optimum, defined by the maximum of the fitness function.

To apply a genetic algorithm to the problem of aligning LC-MS/MS and LC-MS datasets using piecewise linear functions, the candidate solutions $c_{i}$ are encoded by their breakpoints:

$$
c_{i}=\left\{\left(x_{i 1}, y_{i 1}\right),\left(x_{i 2}, y_{i 2}\right) \ldots\left(x_{i n}, y_{i n}\right)\right\}
$$

where $x_{i j}$ is the breakpoint's retention time (or scan number) in the LC-MS/MS dataset and $y_{i j}$ the corresponding retention time in the LC-MS dataset. These breakpoints are sorted to define a continuous and monotonously increasing piecewise linear function. For algorithmic simplicity, $n$ is constant and $x_{i j}=-1$ used to denote a non-existent breakpoint.

A simple fitness function $F\left(c_{i}\right)$ that is inherently insensitive to outliers is the sum over all $N$ matched peptides of the likelihood, or a function proportional to the likelihood, of observing peptide $p$ in the LC-MS dataset at retention time $y_{p}$ if the piecewise linear function evaluates to $y\left(x_{p}\right)$ :

$$
F\left(c_{i}\right)=\sum_{p=1}^{N} e^{-\frac{\left(y_{p}-y\left(x_{j}\right)\right)^{2}}{2 v^{2}}}-k n_{i}
$$

where $\sigma^{2}$ is the residual variance of the retention time in the LC-MS dataset relative to the alignment, either estimated by the program (default) or provided by the user, $n_{i}=\#\left\{\left(x_{i j}, y_{i j}\right) \in c_{i} \mid x_{i j}>0\right\}$ the number of breakpoints in chromosome $c_{i}$ and $k>0$ the uniform cost for each breakpoint. The residual variance is automatically estimated as the standard deviation from the median of the difference between the retention times in the LC-MS dataset for two consecutively eluting peptides in the LC-MS/MS dataset.

In each generation, the half of the chromosomes in the population with lowest fitness is replaced by copies of the half of the chromosomes with the highest fitness, applying three types of mutations: insertion (randomly adding a breakpoint anywhere in the alignment interval), deletion (randomly removing a breakpoint by setting $x_{i j}$ to -1 ), and shifting a breakpoint by small random amount in both dimensions. The insertion and deletion frequencies were held constant at 0.1 per breakpoint and generation. The shift mutation frequency was increased linearly from 0 to 0.5 per breakpoint and generation over the run to reduce the risk of the population adapting to a local optimum early in the run. The genetic algorithm was run for 3000 generations with a population size of 300 chromosomes and a maximum number of 12 breakpoints. In general, the value of $\sigma$ depends on the scale and quality of the chromatography. A value of 10 was used for the FTICR data presented here, spanning 1000 to 2000 scans. The small random shifts were uniformly distributed in $([-30,30],[-10,10])$ and the cost per breakpoint, $k$, was set to 0.5 . These parameters were set a priori and used for all alignments without adjustment. After a single pass through the GA, the solution of the highest fitness was chosen as the alignment of the two datasets.

\section{Results and Discussion}

The alignment method operates on one LC-MS and one LC-MS/MS dataset, using full profile data or peak lists from the LC-MS data and identified peptides in the LC-MS/MS! data! (Figure!1).! The! algorithm! performed well on all experimental data used to evaluate it, including clearly non-ideal datasets such as those shown!in!Figure!1.!The!alignment!algorithm!found!the correct trend in the presence of many incorrectly matched!features!in!otherwise!simple,!linear!cases!(Figure! 2),! and! also! in! non-ideal! (nonlinear)! situations (Figure!3).!The!robustness!of!a!regression!method!can!be qualified! by! Hampel's! breakdown! point! [18],! or (roughly) up to what fraction of outliers a regression always gives some information on the true distribution. The higher the breakdown point, the more robust the regression method. For example, standard least-squares regression is sensitive to outliers and has a breakdown point of 0 . The robust least median squares regression has! a! breakdown! point! of! 0.5! [10].! If! all! outliers! fall along a continuous piecewise linear function, then the breakdown point for the method described here would be close to 0.5 , the point at which candidates fitting true and false matches would have similar fitness. More realistically, if the outliers are randomly distributed, the breakdown point is much closer to 1 (infinite robust- 

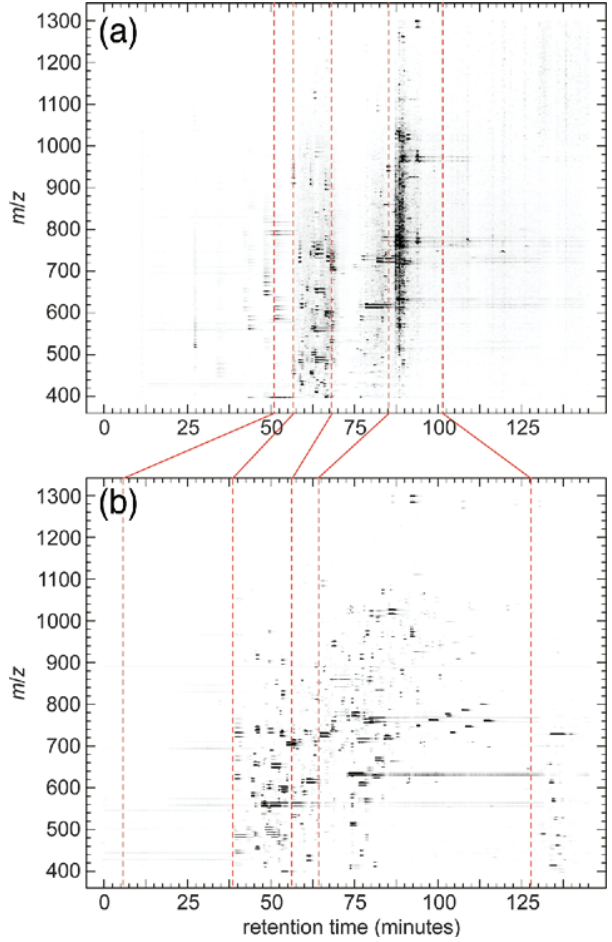

Figure 1. The panels show HCT ion trap LC-MS/MS (a) and FTICR LC-MS (b) data (Gradient 1) from an in-gel tryptic digest of a band from $A$. thaliana total extract run on SDS-PAGE, with the alignment breakpoints shown by the dashed lines. These datasets derive from the slice containing the abundant protein $\mathrm{RuBisCO}$ ( $\sim 30 \%$ !of!the!total!protein!in!green!plant!material![23],!resulting!'in overloading in the chromatographic system. The presented algorithm can align even highly non-ideal datasets such as these. The datasets from other gel slices produced a more linear alignment. The!Pep3D!tool![24]!was!used!to!plot!LC-MS/MS!and!LC-MS!data in mzXML.

ness). For instance, the genetic algorithm still found the main segment of the true distribution when adding 225 random data points in $([0,7000],[0,1500])$ to the 25 data points! in! Figure! 3b! (90\%! outliers)! when! a! reasonable

Figure 2. The algorithm was validated using data not available during development and initial testing; here showing alignments of one HCT ion trap LC-MS/MS dataset to three different FTICR LC-MS datasets (a), (b), and (c) of the same yeast whole cell extract (both Gradient 1). The correctly matched features are narrowly distributed around a trend line, but a significant number of incorrectly matched features are also present. Despite these outliers being far from symmetrically distributed, the genetic algorithm found the linear trend (solid line) of the correctly matched features, inserting 2, 3, and 1 breakpoints in (a), (b), and (c), respectively.
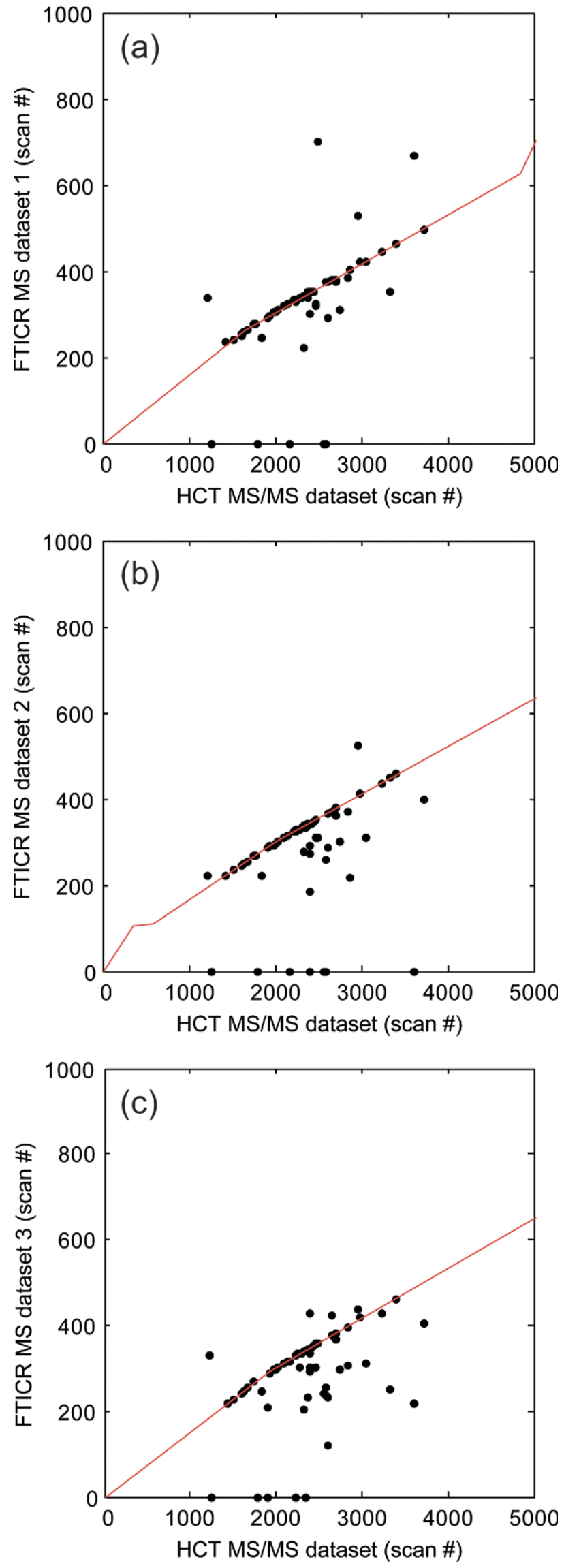

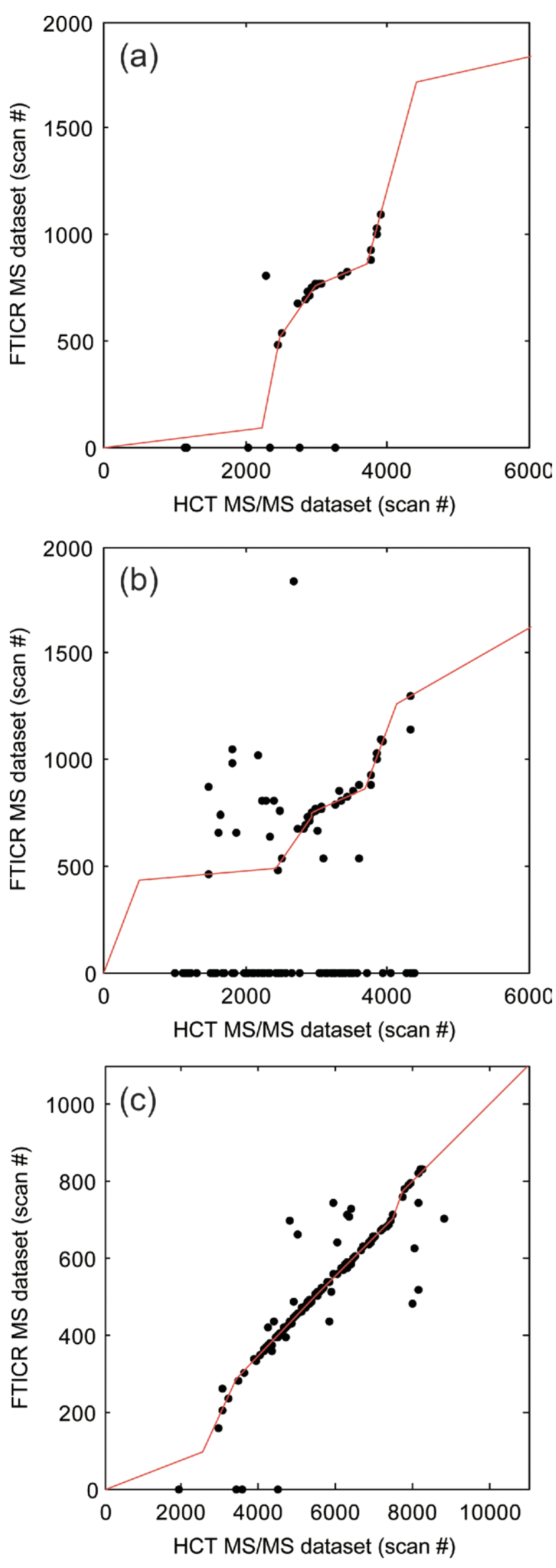

Table 2. The number of unique Mascot protein identifications from four ion trap datasets

\begin{tabular}{lccc}
\hline $\begin{array}{l}\text { LC-MS/MS } \\
\text { dataset }\end{array}$ & $\begin{array}{c}\text { Ion trap } \\
(\mathrm{I})\end{array}$ & $\begin{array}{c}\text { Hybrid } \\
(\mathrm{H})\end{array}$ & $\begin{array}{c}\text { Combined } \\
(\mathrm{I}+\mathrm{H})\end{array}$ \\
\hline \hline 1 & 44 & 44 & $55(+25 \%)$ \\
2 & 75 & 74 & $88(+17 \%)$ \\
3 & 60 & 54 & $68(+13 \%)$ \\
4 & 47 & 45 & $52(+11 \%)$ \\
\hline
\end{tabular}

value on the residual standard deviation (20 scans) after alignment, i.e., the distribution of the true matches along the alignment function, was provided by the user. For data of good quality, or at least 20 peptides distributed throughout the run and no more than $20 \%$ outliers, the program is both fully automatic and robust. The algorithm and automatic determination of residual standard deviation were further validated on 12 pairs of LC-MS and LC-MS/MS datasets (see Supplementary Material section which can be found in the electronic version of this article).

To evaluate the utility of merging ion trap MS/MS and FTICR MS data for protein identification using chromatographic alignment, we used four consecutive ion trap LC-MS/MS analyses of a yeast whole cell digest, analyzed two weeks apart from a separate aliquot of the same digest in a second but identical LC system coupled to the FTICR instrument. Approximately $75 \%$ of the MS/MS spectra from multiply charged precursors could be associated with accurate precursor ion masses from the FTICR data after chromatographic alignment. The average total number of unique protein hits was somewhat lower in the hybrid (H;!see!Table!2)!than!the!ion!trap!(I)!datasets,!likely!due to a combination of incorrectly matched precursors and the restrictive peptide tolerance $(5 \mathrm{ppm})$ used in the Mascot search of hybrid data. However, by combining the two searches $(\mathrm{I}+\mathrm{H})$, the number of unique protein identifications increased $11 \%$ to $25 \%$. We did not observe any significant difference in average size or charge of the identified peptides. However, as expected, the protein identifications were based on fewer peptides per protein in the hybrid dataset. The actual peptide ion score in Mascot is independent of the set tolerance or measurement error of the precursor ion mass, but the ion score required for homology or identity scales inversely with tolerance (mass accuracy). This explains why a number of peptides and proteins

Figure 3. Alignment of HCT LC-MS/MS datasets to FTICR LC-MS datasets of $A$. thaliana SDS-PAGE fractions: (a) the gel band dominated!by!the!abundant!RuBisCO!protein!shown!in!Figure!1. The genetic algorithm finds the correct features even when introducing additional noise by lowering the Mascot ion score cutoff from 30 to 15 (b). Panel (c) shows the piecewise linear fit to an alignment of datasets from an A. thaliana total extract digest (Gradient 2), where most of the correctly matched features fall along a line, with a few clearly offset from this line after a discrete shift near the end of the run. 

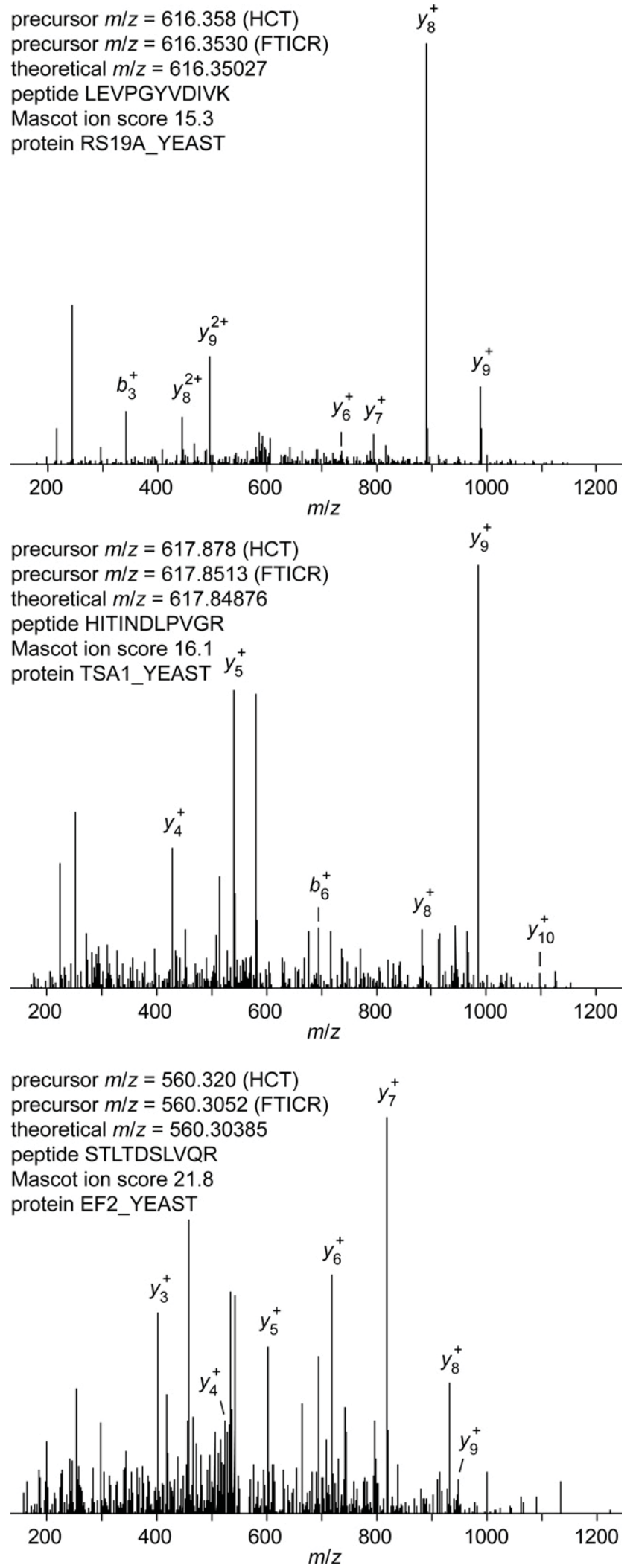

Figure 4. Three ion trap tandem mass spectra with insufficient Mascot ion scores for peptide identification using ion trap data alone, but successfully identified using accurate precursor ion masses following chromatographic alignment.

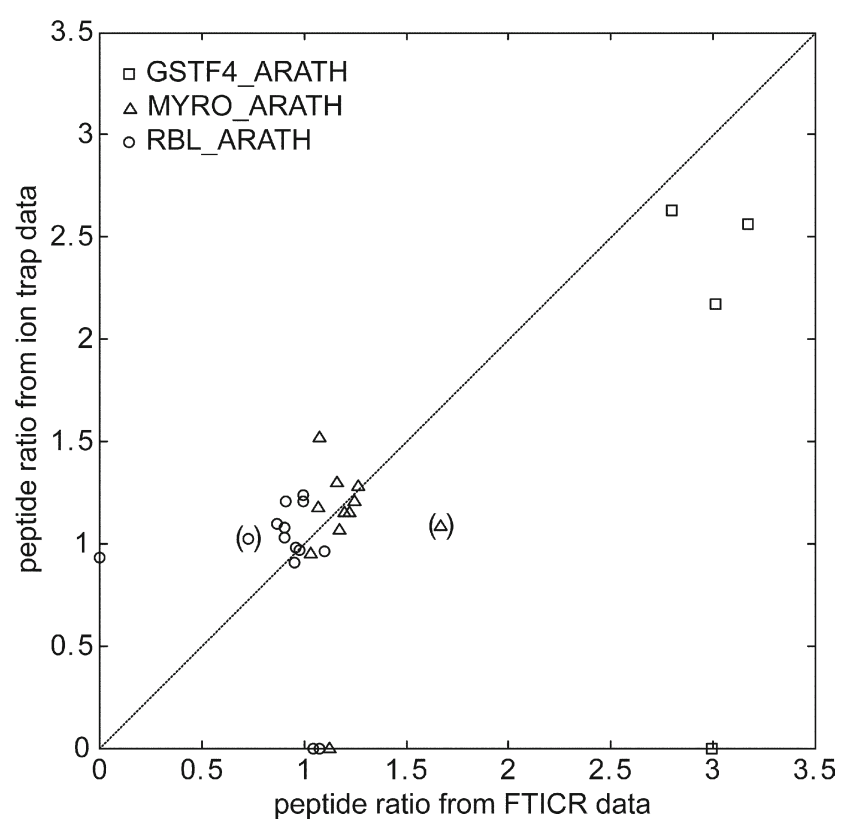

Figure 5. Peptide ratios determined from FTICR and ion trap data for three $A$. thaliana proteins in leaf material from oxidatively stressed plants versus controls: RuBisCO (RBL_ARATH), myrosinase (MYRO_ARATH), and glutathione S-transferase PM24 (GSTF4_ARATH). A peptide ratio value of zero indicates that a peptide was identified in one dataset but not the other.

that could not be confidently identified using the ion trap data alone, could be identified when the ion trap data were combined with accurate precursor ion masses from the FTICR and searched using a much smaller peptide mass tolerance. Three examples of such ion trap MS/MS! spectra! are! shown! in! Figure! 4.! All! three! peptides were identified with accurate precursor ion mass but not from ion trap data alone. The corresponding proteins are abundant in yeast whole cell extracts. RS19A_YEAST is a 40S ribosomal protein, and peroxiredoxin TSA1 (TSA1_YEAST) and elongation factor 2 (EF2_YEAST) are both among the 62 proteins identified from! the! SWISS-2DPAGE! S. cerevisiae gel! (http:// expasy.org/swiss-2dpage).

In the larger $A$. thaliana proteomics experiment, the total protein extract was first separated by SDS-PAGE, and the proteins in each of 25 gel slices digested and analyzed by ion trap LC-MS/MS and FTICR LC-MS using Gradient 1. The ion trap LC-MS/MS and FTICR LC-MS datasets were pairwise chromatographically aligned for each gel slice. Requiring a minimum of two distinct peptides (not counting ${ }^{14} \mathrm{~N}$ and ${ }^{15} \mathrm{~N}$ variants) having ion scores larger than or equal to the identity score for protein identification, equivalent to removing single peptide hits from the Mascot results, the number of significant protein hits increased from 364 in the ion trap data to 432 in the combination with the hybrid dataset with accurate precursor ion masses from FTICR MS data. The hybrid data by itself produced 320 significant protein hits. The number of hits from the random database using the same database search and protein 
identification criteria were 0,1 and 2 for the ion trap, hybrid, and combined datasets respectively, i.e., a false positive rate $<1 \%$ in all datasets.

The combination of ion trap LC-MS/MS and FTICR LC-MS data can also be used in quantitative proteomics where the quantitative information is extracted from the MS data. There are several potential benefits of using FTICR MS over ion trap data for relative protein quantitation. The higher resolution can be used to resolve species of similar $\mathrm{m} / \mathrm{z}$ interfering in low-resolution data. Such interferences lead to larger errors and hence a larger uncertainty if a protein is differentially expressed or not. The larger dynamic range and relative ease by which peaks are separated from background in an FTICR spectrum is beneficial for obtaining more accurate numerical values on very large and very small ratios.!Figure!5!illustrates!some!of!these!points!for!three example proteins in the experiment using metabolic ${ }^{15} \mathrm{~N}$-labeling to study the effect of oxidative stress in $A$. thaliana (this study will be published in its entirety elsewhere).! For! the! ubiquitous! RuBisCO! protein,! the mean ratios and standard deviations were $0.97 \pm 0.07$ and $1.05 \pm 0.11$ for FTICR and ion trap data, respectively, and for the potentially subtly induced myrosinase![19,!20],!the!ratios!were!1.15!+_!0.08!and!1.19!_!0.15 for FTICR and ion trap data, respectively. These values are after removal of two data points in the FTICR data more than two standard deviations from the mean (indicated! by! parentheses! in! Figure! 5).! The! increased abundance of myrosinase relative to RuBisCO is barely significant in the ion trap data, with a two-sided $t$-test $P$-value of 0.9551. In the FTICR data, the corresponding $P$-value is 0.9997 (before removing the outliers). For the strongly! induced! glutathione! S-transferase! [21],! the ratios were $2.99 \pm 0.15$ and $2.46 \pm 0.24$. The correlation between FTICR and ion trap measurements for the same peptide is very weak, suggesting that most of the uncertainty derive from random measurement errors. The systematic difference in the glutathione S-transferase ratios indicates a smaller practical linear dynamic range using the ion trap, though the numerical values for very large or very small ratios may be of little importance if the scope of the experiment is limited to identify differentially abundant proteins. The peptide ratios were extracted from the!ion!trap!data!by!XPRESS![22]!with!default!settings,!and from the FTICR data by a similar program written inhouse, using peak maximum values in a $\pm 10 \mathrm{ppm}$ window and integrating a range from four FTICR spectra before and six spectra after chromatographic maxima to mimic XPRESS while taking advantage of the high mass accuracy and resolving power.

As FTICR mass spectrometry was exclusively used to acquire accurate mass LC-MS data for this work, it was possible to rely on external $\mathrm{m} / \mathrm{z}$ calibration. However, it is conceivable that time-of-flight mass spectrometry may also be used to generate accurate mass data to construct hybrid datasets. In this case, it may be necessary to internally calibrate the spectra to achieve sufficient mass accuracy. In the absence of other reference compounds, the peptides identified in the LC-MS/MS dataset may be used as potential internal calibrants, as previously! shown! for! FTICR! [15]! and! similar! to! the method of!Jaitly!and!coworkers![9].

The! feature extraction and chromatographic alignment were fast, $\sim 30 \mathrm{~s}$ per pepXML and $m z X \mathrm{XML}$ file on a $3.0 \mathrm{GHz}$ Pentium 4 with $2 \mathrm{GiB}$ RAM using line spectra from the FTICR. The conversion of FTICR data took $\sim 20 \mathrm{~min}$ to line spectra $m z \mathrm{XML}$ per $4 \mathrm{GiB}$ LC-MS dataset and was the most time-consuming step in the analysis pipeline. The software is only tested with line spectra $m z X M L$, which is sufficient for protein identification, though the use of full spectral profile data may be beneficial in quantitation, particularly from low signal-to-noise data. The genetic algorithm was designed for simplicity rather than performance, but as it aligns two datasets in less than a minute on a standard desktop PC, it was considered sufficiently fast for all conceivable purposes.

\section{Conclusions}

Alignment of LC-MS and LC-MS/MS datasets is feasible by fitting a piecewise linear function to common features using a robust genetic algorithm. Chromatographic alignment has many potential applications, including improving protein identification and quantitation by combining data from a fast ion trap and a high resolving power instrument such as an FTICR. Separate optimization of chromatographic separation and data acquisition in the two systems may produce an even larger improvement than demonstrated here. Particularly LC-MS/MS benefits from longer chromatographic gradients, and since this data is acquired on comparatively inexpensive instruments, the best price/performance ratio may be achieved matching several such ion traps with one more expensive high resolving power instrument used in LC-MS mode with!shorter!gradients.

The! alignment!program,!msalign,! is freely! available as open source under the GNU General Public License at http://www.ms-utils.org/msalign,! including! a! utility!"hybridMGF”!to!generate!hybrid MGF peak lists for Mascot and other search engines.

\section{References}

1. Li, X. J.; Yi, E. C.; Kemp, C. J.; Zhang, H.; Aebersold, R. A Software Suite for the Generation and Comparison of Peptide Arrays from Sets of Data Collected by Liquid Chromatography-Mass Spectrometry. Mol. Cell. Proteom. 2005,4, 1328-1340.

2. Syka, J. E.; Marto, J. A.; Bai, D. L.; Horning, S.; Senko, M. W.; Schwartz, J. C.; Ueberheide, B.; Garcia, B.; Busby, S.; Muratore, T.; Shabanowitz, J.; Hunt, D. F. Novel Linear Quadrupole Ion Trap/FT Mass Spectrometer: Performance Characterization and Use in the Comparative Analysis of Histone H3 Post-Translational Modifications. J Proteome Res. 2004,3, 621-626.

3. Bylund, D.; Danielsson, R.; Malmquist, G.; Markides, K. E. Chromatographic Alignment by Warping and Dynamic Programming as a Pre-Processing Tool for PARAFAC Modeling of Liquid Chromatography-Mass Spectrometry Data. J Chromatogr. A 2002,961, 237-244.

4. Prakash, A.; Mallick, P.; Whiteaker, J.; Zhang, H.; Paulovich, A.; Flory, M.; Lee, H.; Aebersold, R.; Schwikowski, B. Signal Maps for Mass Spectrometry-Based Comparative Proteomics. Mol. Cell. Proteom. 2006,5, 423-432. 
5. Prince, J. T.; Marcotte, E. M. Chromatographic Alignment of ESI-LC-MS Proteomics Data Sets by Ordered Bijective Interpolated Warping. Anal. Chem. 2006,78, 6140-6152.

6. van Nederkassel, A. M.; Daszykowski, M.; Eilers, P. H.; Heyden, Y. V. A Comparison of Three Algorithms for Chromatograms Alignment. I Chromatogr. A 2006,1118, 199-210.

7. Sadygov, R. G.; Maroto, F. M.; Huhmer, A. F. ChromAlign: A Two-Step Algorithmic Procedure for Time Alignment of Three-Dimensional LC-MS Chromatographic Surfaces. Anal. Chem. 2006,78, 8207-8217.

8. Listgarten, J.; Emili, A. Statistical and Computational Methods for Comparative Proteomic Profiling Using Liquid Chromatography-Tandem Mass Spectrometry. Mol. Cell. Proteom. 2005,4, 419-434.

9. Jaitly, N.; Monroe, M. E.; Petyuk, V. A.; Clauss, T. R.; Adkins, J. N.; Smith, R. D. Robust Algorithm for Alignment of Liquid Chromatography-Mass Spectrometry Analyses in an Accurate Mass and Time Tag Data Analysis Pipeline. Anal. Chem. 2006,78, 7397-7409.

10. Rousseeuw, P. J. Least Median of Squares Regression. J Am. Stat. Assoc. 1984,79, 871-880.

11. Pedrioli, P. G.; Eng, J. K.; Hubley, R.; Vogelzang, M.; Deutsch, E. W.; Raught, B.; Pratt, B.; Nilsson, E.; Angeletti, R. H.; Apweiler, R.; Cheung, K.: Costello, C. E. Hermjakob, H.; Huang, S.; Julian, R. K.; Kapp, E. McComb, M. E.; Oliver, S. G.; Omenn, G.; Paton, N. W.; Simpson, R. Smith, R.; Taylor, C. F.; Zhu, W.; Aebersold, R. A Common Open Representation of Mass Spectrometry Data and Its Application to Proteomics Research. Nat. Biotechnol. 2004,22, 1459-1466.

12. Keller, A.; Eng, J.; Zhang, N.; Li, X. J.; Aebersold, R. A Uniform Proteomics MS/MS Analysis Platform Utilizing Open XML File Formats. Mol. Syst. Biol. 2005,1, 2005

13. Andreev, V. P.; Li, L.; Rejtar, T.; Li, Q.; Ferry, J. G.; Karger, B. L. New Algorithm for $15 \mathrm{~N} / 14 \mathrm{~N}$ Quantitation with LC-ESI-MS Using an LTQ-FT Mass Spectrometer. J. Proteome Res. 2006,5, 2039-2045.
14.! Seattle! Proteome! Center! (SPC)—Proteomics! Tools.! http://tools.proteomecenter.org/software.php

15. Palmblad, M.; Bindschedler, L. V.; Gibson, T. M.; Cramer, R. Automatic Internal Calibration in Liquid Chromatography/Fourier Transform Ion Cyclotron Resonance Mass Spectrometry of Protein Digests. Rapid Commun. Mass Spectrom. 2006,20, 3076-3080.

16. Pruess, M.; Kersey, P.; Apweiler, R. The Integr8 Project-A Resource for Genomic and Proteomic Data. In Silico Biol. 2005,5, 179-185.

17.! The!Cygwin!homepage.!http:/ / www.cygwin.com.

18. Hampel, F. R. A General Qualitative Definition of Robustness. Ann. Math. Stat. 1971,42, 1887-1896.

19. Xue, J.; Jorgensen, M.; Pihlgren, U.; Rask, L. The Myrosinase Gene Family in Arabidopsis thaliana: Gene Organization, Expression, and Evolution. Plant Mol. Biol. 1995,27, 911-922.

20. Kliebenstein, D. J.; Kroymann, J.; Mitchell-Olds, T. The GlucosinolateMyrosinase System in an Ecological and Evolutionary Context. Curr. Opin. Plant Biol. 2005,8, 264-271.

21. Dixon, D. P.; Lapthorn, A.; Edwards, R. Plant Glutathione Transferases. Genome Biol. 2002, 3, Reviews 3004.

22. Han, D. K.; Eng, J.; Zhou, H.; Aebersold, R. Quantitative Profiling of Differentiation-Induced Microsomal Proteins Using Isotope-Coded Affinity Tags and Mass Spectrometry. Nat. Biotechnol. 2001,19, $946-$ 951.

23. Parry, M. A.; Andralojc, P. J.; Mitchell, R. A.; Madgwick, P. J.; Keys, A. J. Manipulation of RuBisCo: The Amount, Activity, Function, and Regulation. J. Exp. Bot. 2003,54, 1321-1333.

24. Li, X. J.; Pedrioli, P. G.; Eng, J.; Martin, D.; Yi, E. C.; Lee, H.; Aebersold, R. A Tool to Visualize and Evaluate Data Obtained by Liquid Chromatography-Electrospray Ionization-Mass Spectrometry. Anal. Chem. 2004,76, 3856-3860. 